\title{
Minimally invasive management of a symptomatic case of Zinner's syndrome: Laparoscopic seminal vesiculectomy and ipsilateral nephroureterectomy
}

\author{
Emanuele Corongiu $^{1}$, Pietro Grande ${ }^{2}$, Valerio Olivieri ${ }^{3}$, Giorgio Pagliarella ${ }^{1}$, Flavio Forte ${ }^{1}$ \\ ${ }^{1}$ Department of Urology, M.G. Vannini Hospital, Rome, Italy; \\ 2 Sorbonne Université, Assistance Publique-Hôpitaux de Paris, Pitié Salpétière, Urology Department, Paris, France; \\ ${ }^{3}$ Department of Urology, Ivrea Hospital - ASL TO 4, Ivrea, Italy.
}

\begin{abstract}
Summary Introduction: Zinner syndrome is a rare developmental anomaly of the Wolffian (mesonephric) duct which is characterized by a triad of obstruction of the ejaculatory duct, the ipsilateral seminal vesicle cyst, and the ipsilateral renal agenesis. Usually is totally asymptomatic, however it can also determine symptoms such as lower urinary tract symptoms, perineal pain, ejaculatory disorders such as painful ejaculation or hematospermia, and infertility. Case report: We present a case of a 51 years old men with a 3-year history of lower urinary tract symptoms, perineal pain, obstructed defecation, recurrent urinary tract infections and infertility. CT scan showed a voluminous cystic neoformation of the left seminal vesicle, hypoplasia of the left kidney and ipsilateral ureteronephrosis. The mass was removed using laparoscopic "en block" seminal vesiculectomy with associated ipsilateral nephroureterectomy. No post-operative complications occurred. At 2-month post-operative control the patient reported an improvement of urinary and rectal symptoms.
\end{abstract}

KEY WORDS: Seminal vesicle cyst; Zinner's syndrome; Nephroureterectomy; Seminal vesciculectomy; Laparoscopy.

Submitted 2018; Accepted 2019

\section{INTRODUCTION}

Zinner syndrome is a very rare condition, first described in 1914 (1), with less than 200 cases reported worldwide, characterized by unilateral renal agenesis, ipsilateral seminal vesicle cyst and ejaculatory duct obstruction. The association between upper urinary tract abnormalities and seminal vesicle malformation are based on the shared origin of the ureteral buds and seminal vesicles from the mesonephric (Wolffian) duct (2). In most cases, these anomalies are completely asymptomatic and the diagnosis is often incidental. However, the association with lower urinary tract symptoms, perineal pain, ejaculatory disorders such as painful ejaculation or hematospermia, and infertility may help unveil the syndrome.

\section{Case report}

We report the case of a 51-year old Caucasian man, presenting with lower urinary tract symptoms (LUTS), perineal pain, obstructed defecation, recurrent urinary tract infections and infertility. Uroflowmetry, showed reduced maximal flow $(9 \mathrm{ml} / \mathrm{s})$, irregular flow pattern and a consistent postvoid residue $(150 \mathrm{ml})$. The evaluation was completed by an abdominal ultrasound which showed a voluminous cystic formation of the left seminal vesicles together with agenesis of the left kidney. Accordingly, CT scan with excretory phase, revealed a small pelvis completely occupied by a voluminous cystic formation dislocating the rectum and the urinary bladder and causing right ureteronephrosis, with ureteral outlet at the level of the cystic mass (Figure 1). Additionally, hypoplasia of the left kidney was noticed (Figure 1). Based on these data, a minimally-invasive surgical approach was planned, as previously reported as a safe and feasible option (3).

A laparoscopic left nefroureterectomy and cystic mass ablation was subsequently planned. The patient was placed in Trendelenburg position: a $12 \mathrm{~mm}$ optical Trocar was inserted $1 \mathrm{~cm}$ above the umbilical fold.

Pneumoperitoneum was obtained and three other trocars (two of 5 and one of $10 \mathrm{~mm}$ ) were placed.

The Douglas fold was completely obliterated by the engorged left seminal vesicle underlying the parietal pelvic peritoneum. The extra-peritoneal space was accessed and the seminal tangle carefully dissected, taking care to avoid direct damages to rectal walls and moreover to the Hovealque plexus located at both lateral rectal sides. Special attention was taken to retract laterally the sacro-recto-genito-pubic ligaments (Delbet bands) in which prostatic and vesical vessels roots are contained. The right ductus deferens was identified and spared, while the left one was necessarily transacted at the point in which it seemed to fuse with the left ureter on the engorged dome of the seminal vesicle, configuring a disturbance of the paramesonephric Wolffian duct development. Once the seminal vesicle removed, the patient was moved in right flank position to perform nephroureterectomy. One more $5 \mathrm{~mm}$ Trocar was inserted at the cross between the pararectal and infracostal left lines. Via detaching the mesosigma and gaining the Jonnesco space, $3-5 \mathrm{~cm}$ above the left common iliac vessels, the aplastic kidney with its small vascular pedicle was found and it was possible to remove the left kidney, the dilated ureter and the cystic mass "en block", with an 
Figure 1.

CT scan.

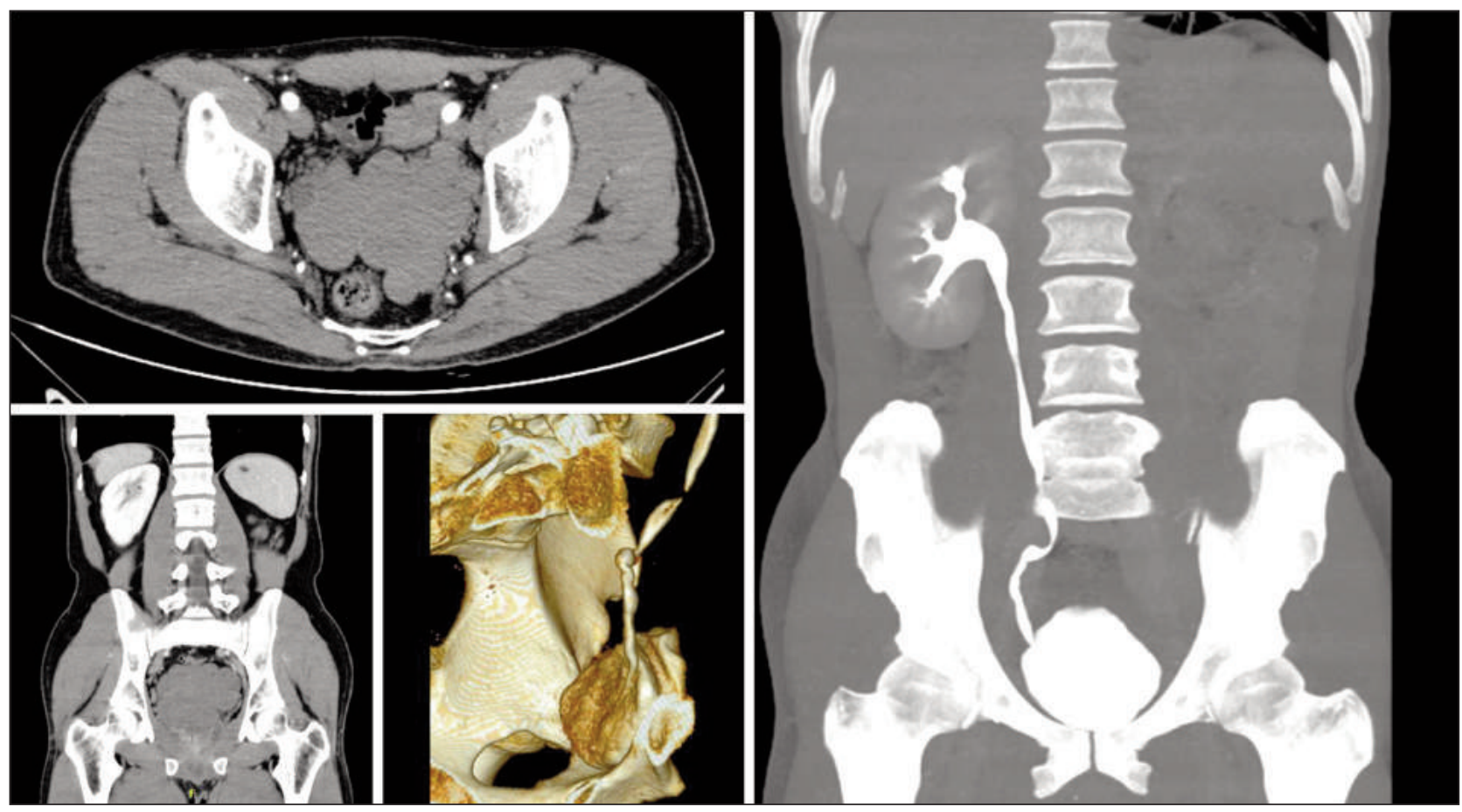

Figure 2.

Hypoplasia of the left kidney and voluminous seminal cystic formation.

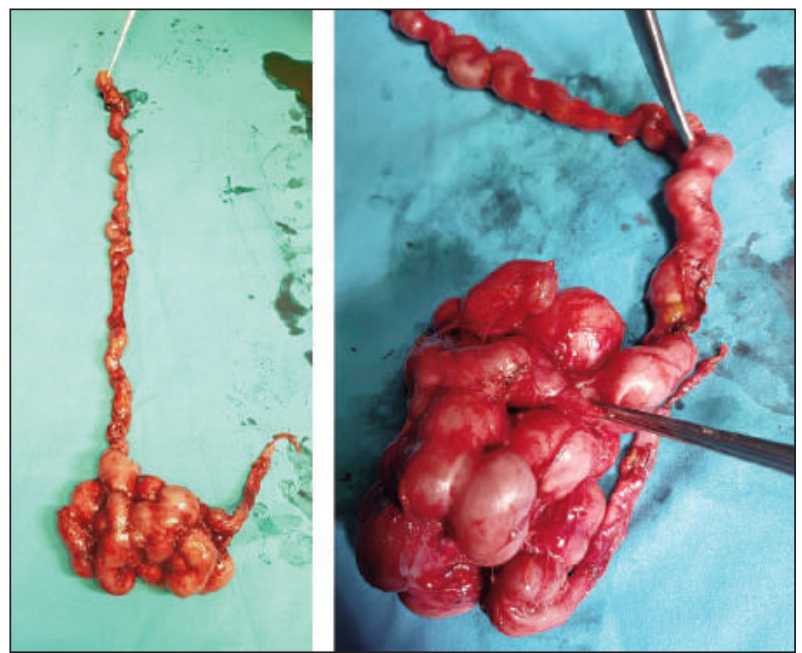

operative time of about 2 hours (Figure 2). The postoperative course was without complications and the patient was discharged on the 4th post-operative day.

\section{Conclusions}

Final pathological analysis revealed a small polycystic left kidney in the absence of residual parenchyma.

The cystic mass (measured at $33 \mathrm{~cm}$ in diameter) was compatible with a polycystic and ectopic seminal vesicle (Figure 2). The patient was subsequently followed-up in outpatient setting, reporting a progressive and continuous improvement LUTS and rectal symptoms.
Approximately 60 days after the surgical procedure, the patient underwent a new uroflowmetric check which showed a more regular pattern and significantly improved urinary indices $\left(Q_{\max } 15 \mathrm{ml} / \mathrm{S}\right)$ and a reduction of post-void volume $(60 \mathrm{cc})$.

\section{REFERENCES}

1. Zinner A. Ein fall von intravesikaler samenblasenzyste. Wein Med Wochenschr. 1914; 64:605-609.

2. Kao CC, Wu CJ, Sun GH, et al. Congenital seminal vesicle cyst associated with ipsilateral renal agenesis mimicking bladder outlet obstruction: a case report and review of the literature. Kaohsiung J Med Sci. 2010; 26:30-4.

3. Kiremit MC, Acar O, Sag AA, et al. Minimally invasive management of Zinner's syndrome with same-session robot-assisted seminal vesiculectomy and ipsilateral nephroureterectomy using a single geometry of trocars, J Endourol Case Rep. 4: 1, 186-189.

\section{Correspondence}

Emanuele Corongiu, MD (Corresponding Author) emanuele.corongiu@libero.it

Giorgio Pagliarella, MD

Flavio Forte, MD

G.M. Vannini Hospital, Department of Urology Via di Acqua Bullicante 4, 00177, Rome (Italy)

\section{Pietro Grande, MD}

Sorbonne Université, Assistance Publique-Hôpitaux de Paris, Pitié Salpétière, Urology Department, Paris (France)

Valerio Olivieri, MD

Department of Urology, Ivrea Hospital - ASL TO 4, Ivrea (Italy) 